\title{
Response of the mosquito protein interaction network to dengue infection
}

\author{
Xiang Guo 1,2,4, Yao Xu1', Guowu Bian¹, Andrew D Pike1, Yan Xie³ and Zhiyong Xi*1
}

\begin{abstract}
Background: Two fifths of the world's population is at risk from dengue. The absence of effective drugs and vaccines leaves vector control as the primary intervention tool. Understanding dengue virus (DENV) host interactions is essential for the development of novel control strategies. The availability of genome sequences for both human and mosquito host greatly facilitates genome-wide studies of DENV-host interactions.

Results: We developed the first draft of the mosquito protein interaction network using a computational approach. The weighted network includes 4,214 Aedes aegypti proteins with 10,209 interactions, among which 3,500 proteins are connected into an interconnected scale-free network. We demonstrated the application of this network for the further annotation of mosquito proteins and dissection of pathway crosstalk. Using three datasets based on physical interaction assays, genome-wide RNA interference (RNAi) screens and microarray assays, we identified 714 putative DENV-associated mosquito proteins. An integrated analysis of these proteins in the network highlighted four regions consisting of highly interconnected proteins with closely related functions in each of replication/transcription/ translation (RTT), immunity, transport and metabolism. Putative DENV-associated proteins were further selected for validation by RNAi-mediated gene silencing, and dengue viral titer in mosquito midguts was significantly reduced for five out of ten (50.0\%) randomly selected genes.

Conclusions: Our results indicate the presence of common host requirements for DENV in mosquitoes and humans. We discuss the significance of our findings for pharmacological intervention and genetic modification of mosquitoes for blocking dengue transmission.
\end{abstract}

\section{Background}

Dengue fever and associated dengue hemorrhagic fever are emerging globally as the most important arboviral disease threatening human populations. Dengue virus (DENV) is transmitted to humans by the mosquitoes Aedes aegypti and Aedes albopictus. When ingested into the mosquito midgut with blood, DENV first interacts with midgut cell membrane receptors and then enters the cells through receptor-mediated endocytosis. Following replication in midguts, DENV disseminates to salivary glands for transmission to human. In the mosquito, DENV is attacked by the mosquito innate immune system, including RNAi and Toll pathway [1,2]. In addition, the effects of several host genes were reported to deter-

* Correspondence: xizy@msu.edu

1 Department of Entomology and Genetics Program, Michigan State University, East Lansing, Michigan 48824, USA

Full list of author information is available at the end of the article mine the mosquito's susceptibility to dengue infection [3].

DENV belongs to the genus Flavivirus of the Flaviviridae family. Flaviviruses are small, enveloped viruses with a single-stranded positive-sense RNA, which can be translated into a single polyprotein by host cell proteins. The polyprotein is then cleaved into individual proteins by both viral and host proteases [4]. Three structural proteins (capsid, pre-membrane, and envelope) constitute the virus particle. Seven non-structural proteins (NS1, NS2A, NS2B, NS3, NS4A, NS4B, NS5), ${ }^{5}$ and 3' untranslated regions (UTR) are involved in RNA replication. The $\mathrm{N}$-terminus of NS3 codes a serine protease essential for virus replication, and the central domain of NS2B is a cofactor for the NS3 serine protease. Virus-host interactions start with binding of the envelope protein $\mathrm{E}$ to a cellular receptor. Then, a complex interplay of viral and cellular proteins modulates the process of viral translation, replication, and assembly [5]. Most previous studies 
on the replication mechanisms of DENV have been conducted in human cells. These studies identified putative cellular receptors and other host proteins that may interact with viral proteins or RNAs [6]. In contrast, relatively little has been done to elucidate how DENV utilizes the cellular factors to enter and replicate in mosquito cells. It remains unclear which virus replication features are responsible for efficient virus passage between these different host systems.

Availability of genome sequences for both humans and the mosquito vector, Ae. aegypti, now provides us novel opportunities to identify the host factors that are required for dengue infection. Recently, microarray and RNAi screens were used for genome-wide studies of interactions between DENV and their hosts. They led to identification of a number of cellular factors that can determine the susceptibility and resistance of both hosts to DENV [2,7]. These results also emphasize the complexity of interactions between flaviviruses and its hosts. Understanding of such interactions requires systematic and comprehensive studies of the host biology as it relates to viral infections. Analysis of protein interaction network is one way to dissect such complex interactions and has been broadly used to study the systems biology of host-pathogen interactions [8-11].

This systems biology approach requires the construction of signaling and regulatory networks. Physical interactions between two proteins are the basis for protein complexes and signaling networks, while transcription factors regulate gene transcription by binding to their target DNA sequences in the context of intricate transcriptional regulatory networks. Large amounts of highthroughput data are now available in several model organisms for protein-protein interactions [12-14], which have been extensively used in network reconstruction and systems biology studies. However, the high cost of experimental methods prevents large scale interaction network mapping of newly sequenced genomes. Computational approaches provide a viable alternative for protein-protein interaction mapping. Experimental interactomes in model organisms can be transferred to other species based on homology information. This interolog approach has been widely used to build protein interaction networks in humans $[15,16]$ and other species [17]. Although unique interactions in a new species can not be found by the interolog method, the predicted interactomes may reveal conserved complexes and pathways, and serve as important tools for annotating uncharacterized proteins [18]. The same concept can be applied to predict interactions between proteins in different species.

Uetz et al. predicted interactions between herpesvirus and human proteins based on known interacting orthologs in model organisms, and co-immuniprecipita- tion confirmed $68 \%$ of their predictions [19]. DENV consists of only 10 proteins, which lack orthologs in model organisms. However, viruses in the genus Flavivirus often target the same host proteins, so known flavivirus-host interactions may be used to predict DENV-mosquito protein interactions. Recently, a high throughput RNAi screen in human cells identified 283 host susceptibility factors and 22 host resistant factors for the infection of West Nile virus (WNV; genus Flavivirus, family Flaviviridae). More importantly, targeted silencing of the WNV host factors found that half of the 283 susceptibility factors and all of the 22 resistant factors affected dengue infection in the same manner as they affected WNV infection [20]. Additional independent studies also provide evidence that multiple flaviviruses, including WNV, Japanese encephalitis (JEV) and DENV, target the same host proteins $[21,22]$. It was reported that these flaviviruses share a similar genomic organization and replication strategy although they cause a range of distinct clinical diseases in humans [6].

Evidence also indicates the notable conservation of required dengue host factors between insects and humans. Recently, a genome-wide RNA interference screen in Drosophila melanogaster cells identified 116 dengue virus host factors. Among them, 82 have human homologues. Using targeted siRNA, $51.2 \%$ of them proved to be dengue host factors in humans [7]. A number of conserved host factors were also identified in several earlier studies. In both mosquitoes and humans, La protein interacts with $5^{\prime}$ and $3^{\prime}$ ends of dengue viral RNA and two nonstructural proteins NS3 and NS5. Such interactions seem important for assembly of a functional replication complex of dengue virus [23,24]. Lamininbinding protein and heat shock proteins were also indicated to mediate the dengue infection in both humans and mosquitoes [21,25-28]. Moreover, a similar internalization route (clathrin-mediated endocytosis) was used by dengue viruses to enter into human and mosquito cells.

In this study, we have developed the mosquito protein interaction network based on large-scale protein interaction datasets in Saccharomyces cerevisiae, Caenorhabditis elegans and Drosophila melanogaster. 714 flavivirus-associated mosquito proteins were inferred from three data sources based on physical interaction assays, genomewide RNAi screens and microarray results. An integrated analysis of dengue-associated protein interaction subnetworks highlighted four regions consisting of highly interconnected proteins with closely related functions. Finally, we verified that five out of ten (50.0\%) randomly selected genes were required for DENV infection in the main mosquito vector, Ae. aegypti, by RNAi-mediated gene silencing. 


\section{Results and Discussion Construction of the first-draft mosquito protein interaction map}

Reliable protein interaction datasets in model organisms are the basis of the interolog method. Because large-scale protein interaction mapping generates a high number of false positive interactions, it is essential to assess the quality of experimentally determined protein interactions. To generate the draft mosquito interaction network, we selected different interaction datasets with available confidence scores or rankings. The dataset in $S$. cerevisiae includes 47,783 interactions identified by coimmunoprecipitation and yeast two-hybrid screens. Each interaction is associated with a confidence score estimated by the logistic regression model [29]. The twohybrid-based dataset in D. melanogaster includes 20,405 interactions with a confidence score estimated by a generalized linear model [12]. The dataset in C. elegans (4,736 interactions) includes literature-mined data and two-hybrid generated data divided into three confidence classes [13]. For all three datasets, confidence metrics were generated using only statistical and topological predictors, such as the number of interaction partners and a local clustering coefficient. These scores were combined with the InParanoid [30] score to generate confidence values for predicted interactions in Ae. aegypti.

Our predictions resulted in 64,449 interactions for 6,263 mosquito proteins, for which the average confidence score is 0.32 (Additional file 1). To test the effectiveness of our confidence scoring scheme, we examined the association of semantic similarity and confidence score for the predicted interactions. Interacting proteins often participate in the same molecular pathway and are located in the same cellular compartment. Functional relatedness has been shown to be the best feature in discriminating true interactions from noise [31], and our previous work demonstrated the advantage of Gene Ontology (GO)-based similarity measures in protein interaction validation [32]. Among our predicted interactions, there are $32,826,36,132$, and 20,127 interacting protein pairs with annotation of three GO sub-categories: biological process, molecular function, and cellular component, respectively. Our results indicate that high confidence interactions correlate with protein pairs with high semantic similarity, suggesting the effectiveness of our confidence scoring scheme and the quality of our predictions (Fig. 1A). Because a large number of GO annotations for Ae. agypti proteins are based on sequence homology to $D$. melanogaster, the high correlation between semantic similarity and confidence score of interacting proteins predicted from Drosophila may simply reflect their correlation in Drosophila. Thus, we have repeated the analysis using interactions predicted from yeast alone. This dataset also shows an apparent correla-

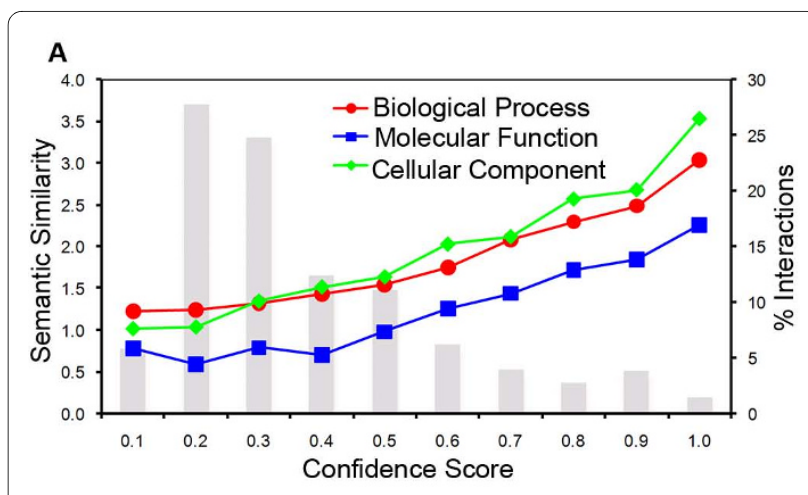

B

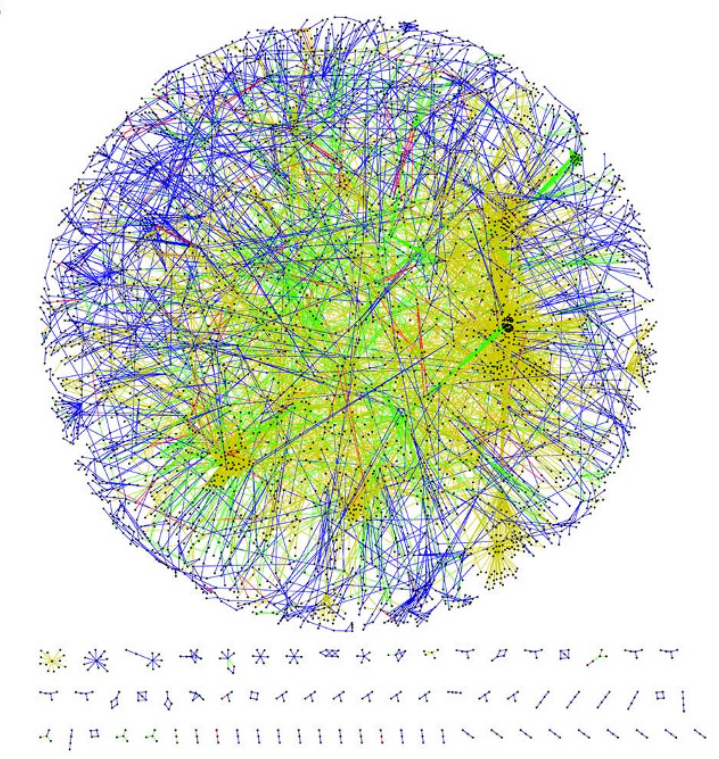

Figure 1 Construction of the mosquito protein interaction network. (A). Semantic similarity and interaction confidence of putative interacting proteins in Ae. aegypti. Protein interactions were binned based on their confidence scores. The average semantic similarity values in each bin were shown for all three GO sub-categories: biological process, molecular function, and cellular component. Percentage of interactions in each bin was shown as a histogram bar. (B) Visualization of the predicted mosquito protein interaction network. Colors are used to show the origin of the inferred interactions. D. melanogaster: blue, C. elegans: green, S. cerevisiae: yellow, multiple sources: red.

tion between interaction confidence and functional similarity (Additional file 2), indicating the effectiveness of our confidence scoring scheme.

Using predicted interactions with confidence values more than 0.5 , we constructed a weighted protein interaction network for Ae. aegypti (Fig. 1B). The network includes 4,214 mosquito proteins with 10,209 interactions, of which $62 \%, 25 \%, 11 \%$ and $2 \%$ were inferred from yeast, fly, worm, and more than one reference organism, respectively (Fig. 1B). Among all the proteins in our network, 3,500 proteins are connected into a single interconnected network by 9,719 interactions. The number of interacting partners per node in the interconnected net- 
work is 5.55 , and the degree distribution fits a power law distribution with a degree exponent of 1.79. As expected, the network also has a high clustering coefficient (0.32) and a short path length (6.46).

\section{Prediction of mosquito protein function}

Cellular functions are often carried out by a group of physically or functionally linked molecules that work together to complete distinct tasks. The high clustering coefficient observed in our network indicates that isolated functional modules may be identified from the network. The Markov Cluster algorithm (MCL) identifies highly connected modules based on simulation of stochastic flow in graphs. It has been shown to be the most robust algorithm for the extraction of protein complexes from protein-protein interaction networks [33]. Using this algorithm, we parsed the mosquito protein interaction network into 494 densely connected clusters (Additional file 3 ).

We searched for GO biological processes overrepresented in clusters with more than 10 members and functionally annotated 78 clusters (Fig. 2 and Additional file 4). The results indicate that $76 \%$ of clusters have one or more enriched GO terms (Benjamini-corrected $\mathrm{p}$ value < 0.05). The composition of each cluster is a valuable resource for the annotation of hypothetical proteins in Ae. aegypti. For example, cluster 14 comprising 26 proteins represents the RNA polymerase complex (inset of Fig. 2). There are eight hypothetical proteins in this cluster while the remaining eighteen proteins are related to transcription with sixteen of them (61.5\%) annotated as DNA-directed RNA polymerase. It is most likely that the eight hypothetical proteins also belong to this functional group. Thus, putative function of hypothetical proteins might be inferred by their proximity to functionally annotated proteins in a cluster. Considering that a large number of Ae. aegypti genes are defined as hypothetical proteins, our clustered interaction network provides an useful resource for further annotation of those genes.

\section{Emerging hypotheses of biological pathways in mosquitoes}

Detailed examination of local interaction networks may reveal interconnections of biological pathways and provide further insights on how a biological process is controlled in mosquitoes. Here we focus on the mosquito biological processes that have attracted increasing interest in recent years.

The mosquito Toll pathway is involved in defense against fungal and viral infection $[2,34]$. Activation of the Toll pathway leads to a cascade of events that result in the degradation of CACT, translocation of REL1 transcription factors to the nucleus, and a rapid increase in synthesis of antimicrobial compounds and other effectors [3537]. As expected, we observed that CACT interacts with both REL1A [VectorBase: AAEL007696] and REL1B [VectorBase: AAEL006930] in the network (Fig. 3). CACT also interacts with AAEL009326, an ortholog of Drosophila Fmr1, along with the hypothetical protein AAEL014957. Interestingly, Fmr1 has been shown to be

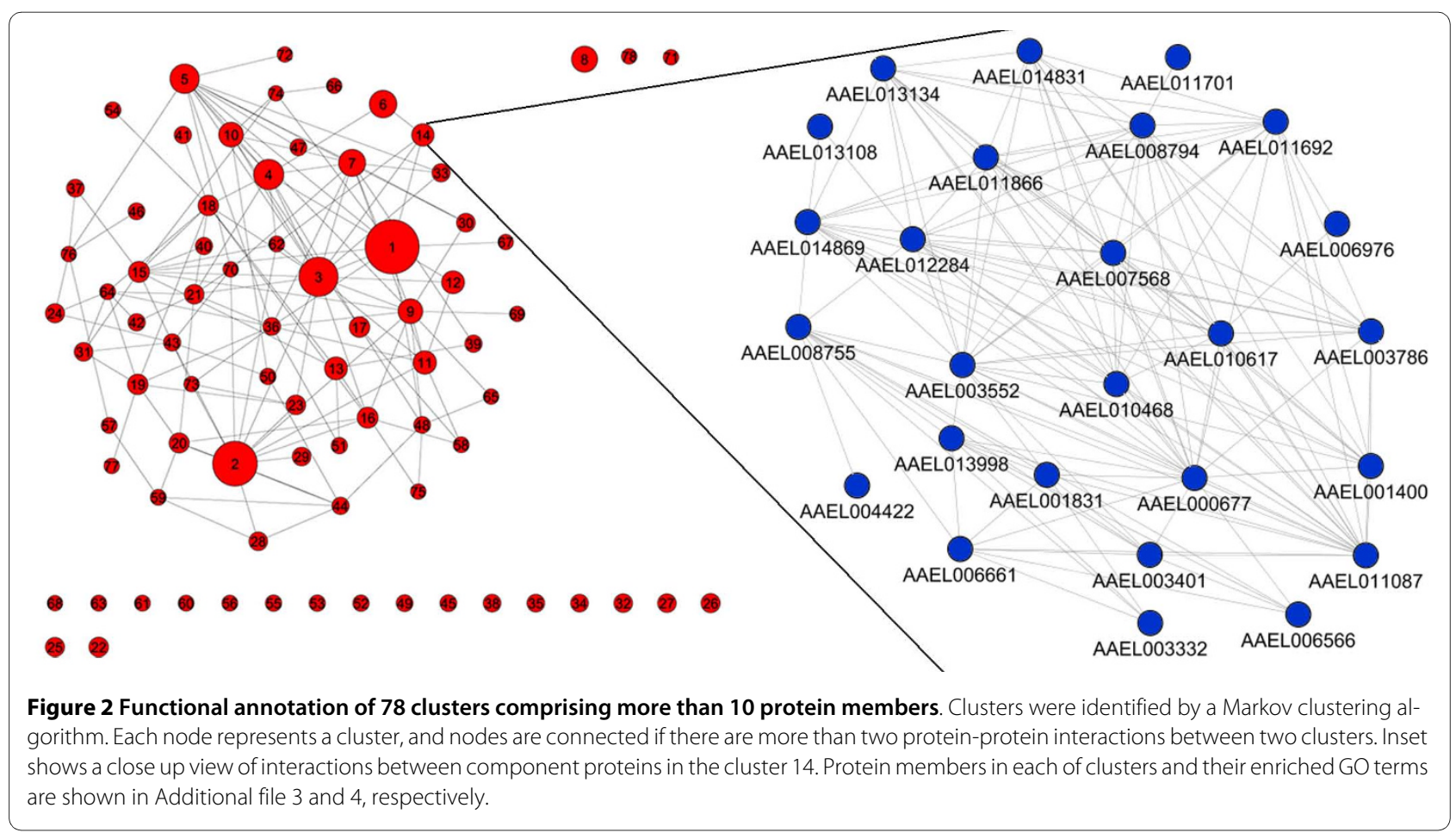




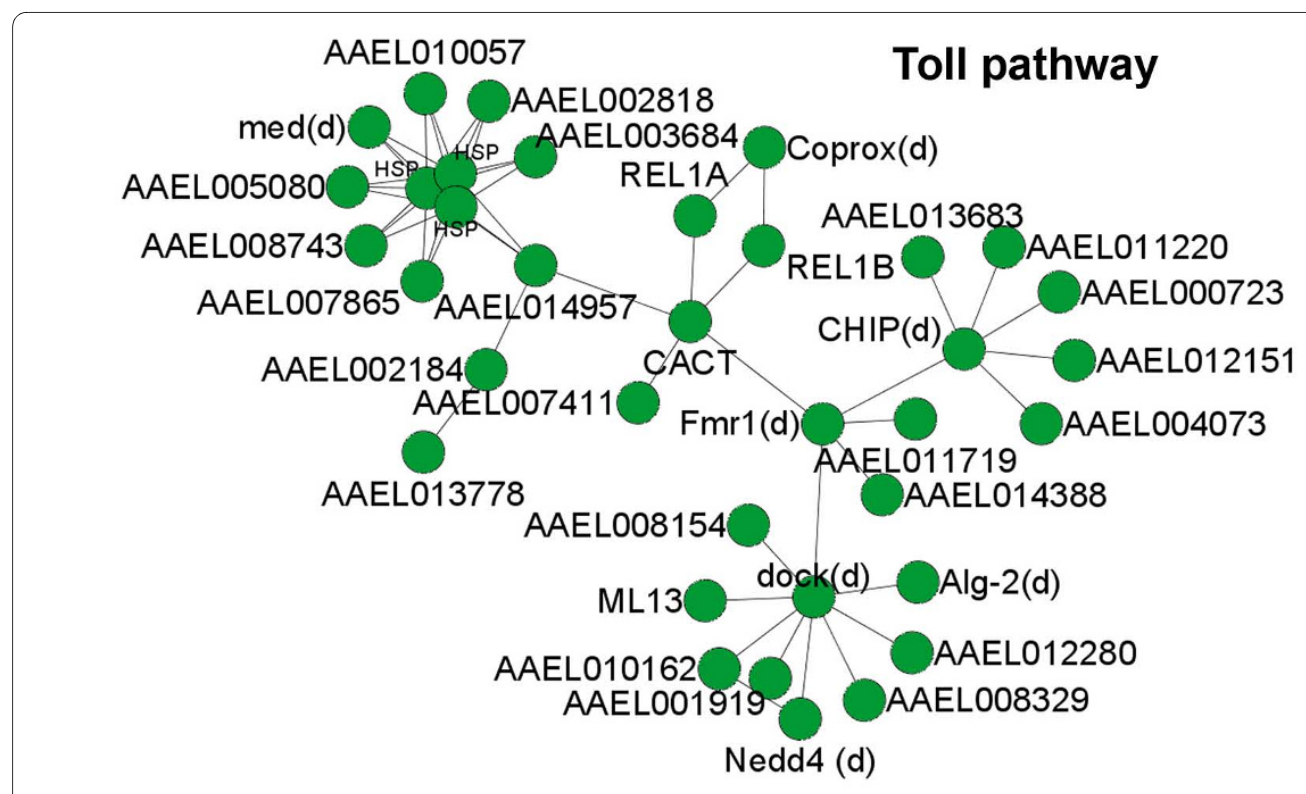

JAK/STAT pathway

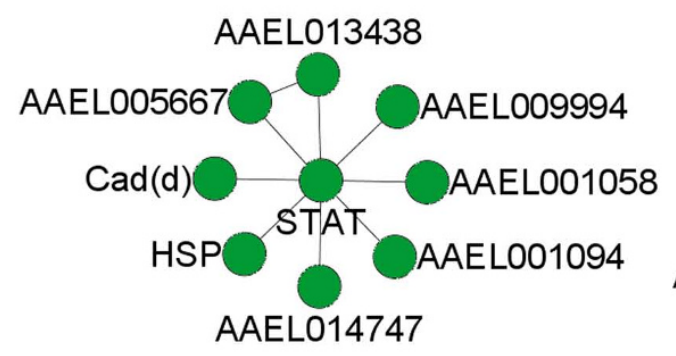

Sex determination

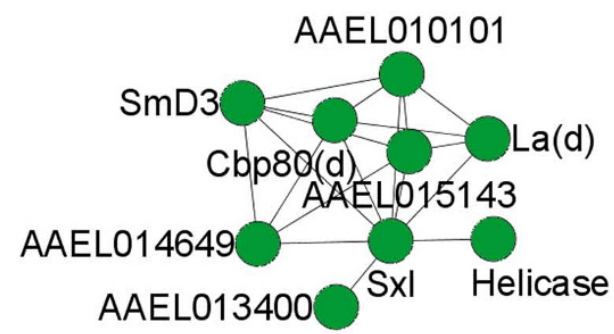

Insulin pathway

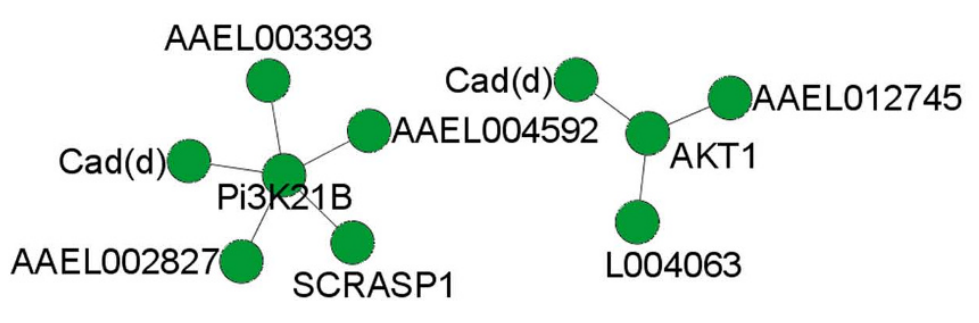

Figure 3 Local views of mosquito pathways or biological processes. Genes related to Toll signaling pathway, JAK/STAT pathway, Insulin pathway, sex determination pathway and mosquito olfaction are selected to identify their interaction partners in the network.

part of the RNAi-related machinery [38]. In Drosophila, Fmr1 forms a complex comprising of Argonaute 2 (AGO2) and a Drosophila homolog of p68 RNA helicase (Dmp68), both of which are required for efficient RNAi [38]. Considering that both the RNAi and Toll pathways can control dengue infection in mosquitoes, it will be worthwhile to test whether Fmr1 serves as a potential link between the two pathways. Moreover, our network indicates that Fmr1 interacts with dock [VectorBase: AAEL013539], an insulin pathway related gene, which further interacts with an apoptosis-related protein AAEL005910 (Drosophila ortholog of Alg-2), an ubiquitin-protein ligase AAEL002536 (nedd-4) and MD2-like receptor AAEL006854 (ML13). In addition, CACT indi- 
rectly interacts with three heat shock proteins (Hsp), including AAEL0011704, AAEL0011708, AAEL014843, and one Hsp70-interacting protein CHIP [VectorBase: AAEL012588] (Fig. 3).

STAT is a key component in the JAK/STAT pathway. In the mosquito protein interaction network, it interacts with eight proteins including AAEL014557, an ortholog of Drosophila caudal (Cad). Cad also interacts with two proteins associated with the Insulin signaling pathway: Pi3K21B [VectorBase: AAEL013596] and AKT1 [VectorBase: AAEL008823] (Fig. 3). This implies a potential link between the JAK/STAT and Insulin pathways. Further examination of the network found Cad interacting with 49 proteins. Thus, as a hub protein in the networks, Cad may have diverse functions and be essential for the survival of Ae. aegypti.

Sex lethal $(\mathrm{Sxl})$ is involved in sex determination in Drosophila. In the network, eight proteins interact with Ae. aegypti Sxl [VectorBase: AAEL011150]. Two of them are hypothetical proteins. Although no evidence suggests these eight proteins are involved in sex determination, six of them are involved in either RNA binding or RNA helicase activity (Fig. 3). Thus, similar to its Drosophila ortholog, Sxl might function as a splicing and translational regulator in mosquito.

Mosquito odorant-binding proteins (Obp) were input into the network to identify their interaction partners. Five odorant-binding proteins and two hypothetical proteins interact with the sterol carrier protein-2 [VectorBase: AAEL002687], a protein which is thought to be involved in intracellular cholesterol transport. OBP56e [VectorBase: AAEL006650] interacts with Hyperkinetic, a gene involved in potassium ion transport, and one hypothetical protein (Fig. 3).

\section{Prediction and analysis of mosquito responses to dengue infection}

A comprehensive mapping of mosquito protein interactions targeted by DENV could provide specific hypotheses and a broad perspective on DENV strategies for replication and persistence in mosquitoes. Previous microarray assays revealed broad responses to DENV infection in mosquitoes that entailed a variety of physiological systems [2]. A recent genome wide RNAi screen in human demonstrated a complex dependence of flaviviruses on host cell physiology, requiring a wide variety of molecules and cellular pathways for successful infection [20]. In addition, studies of physical interactions between flaviviruses and their hosts also provided insight on how viruses hijack the host pathways for their entry and replication. With the mosquito protein interaction network constructed, we can now put all these together and perform an integrated analysis to identify the spectrum of mosquito pathways activated by DENV and other flaviviruses.

\section{Prediction of dengue-associated Ae. aegypti proteins}

We inferred Ae. aegypti proteins associated with DENV infection using three separate datasets. The first dataset was based on RNAi screens in human and Drosophila cell lines. Among 82 DENV host factors identified in Drosophila with recognizable human homologues, 42 are also human DENV host factors [7]. In addition, 305 human genes have been shown to be associated with WNV, of which 124 also affect DENV infection [20]. Given these virus-associated host genes, we inferred 243 Ae. aegypti orthologs which may be involved in DENV infection (Additional file 5). These included 46, 168 and 29 Ae.aegypti orthologs from DENV host factors in Drosophila, human and both, respectively. Secondly, we predicted 22 mosquito proteins that may directly bind to flavivirus proteins or RNAs based on known host-flavivirus physical interactions collected by literature search (Additional file 6). The third dataset was derived by genome-wide transcriptional profiling of Ae. aegypti response to dengue infection. A large number of genes $(n$ $=456$ ) in mosquito midgut and the remaining carcass are differentially expressed in response to virus infection [2]. Taken together, we have identified 714 putative dengue associated-mosquito proteins.

There is an overlap between the three datasets with 7 proteins supported by two data resources (Table 1). The latter includes two putative immune related genes: CTLMA12 [VectorBase: AAEL011455] and RM62A [VectorBase: AAEL001317]. CTLMA12 is a member of the gene family C-Type Lectins (CTLs), which are host pathogen-recognition receptors that are specialized in sensing invading pathogens [39]. RM62A is involved in RNAi process http://cegg.unige.ch/Insecta/immunodb, which has been shown to an important pathway through which mosquito defend against dengue infection.

We identified enriched GO terms from the three datasets based on a hypergeometric test with Benjamini and Hochberg multiple testing correction (Table 2). Strikingly, we observed that the same biological processes were overrepresented in RNAi and physical interactioninferred datasets (Table 2). Two GO terms, protein transport and establishment of localization, were significantly enriched in putative mosquito proteins that bind to viral proteins/RNAs and affect the viral infection $(\mathrm{p}<0.05)$. To initiate infection, DENV binds to and enters host cells via receptor-mediated endocytosis [40]. Physical interaction between the dengue $\mathrm{E}$ protein and the mosquito tubulin protein [VectorBase: AAEL002851] has been shown to facilitate internalization of DENV [41], and the RNAi screen provided additional evidence for the role of tubulin in the assembly/transport of the virus particles. DENV 
Table 1: Putative DENV-associated Ae. aegypti proteins supported by more than one experimental evidences

\begin{tabular}{ccccc}
\hline Gene ID & Gene name or description & Physical Interaction & Microarray & RNAi \\
\hline AAEL001317 & RM62A & + & - & $+($ WNV $)$ \\
AAEL002851 & tubulin beta chain & + & - & $+($ WNV $)$ \\
AAEL001405 & clathrin coat assembly protein & N/A & + & $+($ WNV\&DENV $)$ \\
AAEL009360 & serine/threonine protein kinase & N/A & + & $+($ WNV $)$ \\
AAEL014368 & sap18 & N/A & + (WNV) \\
AAEL011455 & CTLMA12 & N/A & $+($ WNV\&DENV) \\
AAEL009565 & conserved hypothetical protein & N/A & + \\
\hline
\end{tabular}

The results are derived from RNAi screen, physical interaction assay and microarray assay. Except indicated in parenthesis, data are from DENV-host interactions. + , interactions supported by experiments; -, interaction not supported by experiments; N/A, data are unavailable; DENV, dengue virus; WNV, West Nile virus.

also utilizes host proteins for the replication of its genome. The minus-strand 3'UTR of DENV might form the replication complex with calreticulin [VectorBase: AAEL001005], which may actively participate in the process of RNA-dependent RNA synthesis [42]. While RNAi screens investigate the role of host genes in viral infections, microarray profiling shows the expression change of host genes in response to viral infections. Cell death was enriched in the microarray dataset, which was consistent with previous studies indicating that DENV infection induced the expression of mosquito genes involved in apoptosis [2] and resulted in mosquito cell death [43].Our results suggest that different pathways may be involved in the life cycle of viruses and host responses to viral infections.

\section{Network analysis of putative dengue-associated mosquito proteins}

Among the 714 putative DENV-associated proteins, 280 proteins could be found in the weighted Ae. aegypti protein interaction network. We created sub-networks associated with DENV infections by including neighbor proteins that directly connect to at least three of the above DENV-associated proteins. After removing isolated proteins and clusters with less than four members, we identified one major and five minor sub-networks, which contain 121 DENV-associated proteins and 129 neighbor proteins connected by 1,842 interactions (Fig.
4). Further functional analysis of the major sub-network highlighted four regions consisting of highly interconnected proteins with closely related functions in each of replication/transcription/translation (RTT), metabolism, immunity and transport (Fig. 4). To confirm that the above functional groups are more interconnected than the rest of the network, we calculated their graph density in comparison with the density of the global network and random protein groups of the same size taken from the same network. The graph density is defined as the ratio of the number of edges and the number of possible edges in the graph. There are 229 proteins connected by 1,826 edges in the major dengue-associated sub-network with a graph density of 0.07. Functional group RTT consists of 133 proteins connected by 1,655 edges with a graph density of 0.19 . Then, we randomly retrieved 133 proteins from the major dengue-associated sub-network and calculated its graph density. Out of 1,000 repeated random sampling, there were only 6 random groups that have graph density more than that of the RTT group, indicating a permutation-based p-value of less than 0.01. Similarly, the graph densities for metabolism, immunity and transport groups were calculated to be $0.14,0.30$ and 0.34 , respectively. Their permutation-based p-values are all less than 0.01, supporting the existence of distinct functional groups in the dengue-associated protein interaction network.

Table 2: Enriched Gene Ontology (GO) biological process terms in three datasets of DENV-associated Ae. aegypti proteins

\begin{tabular}{lccc}
\hline GO Terms & RNAi* & Physical Interaction* & Microarray* \\
\hline Establishment of Localization & $5.0 \mathrm{E}-4$ & $1.3 \mathrm{E}-2$ & NS \\
Protein Transport & $2.4 \mathrm{E}-2$ & $1.3 \mathrm{E}-2$ & NS \\
Response to stimulus & NS & NS & $4.4 \mathrm{E}-3$ \\
Cell death & NS & NS & $5.7 \mathrm{E}-2$ \\
\hline
\end{tabular}

Only P values that reach the significant level are shown. ${ }^{*} \mathrm{NS}$, non-significant. 


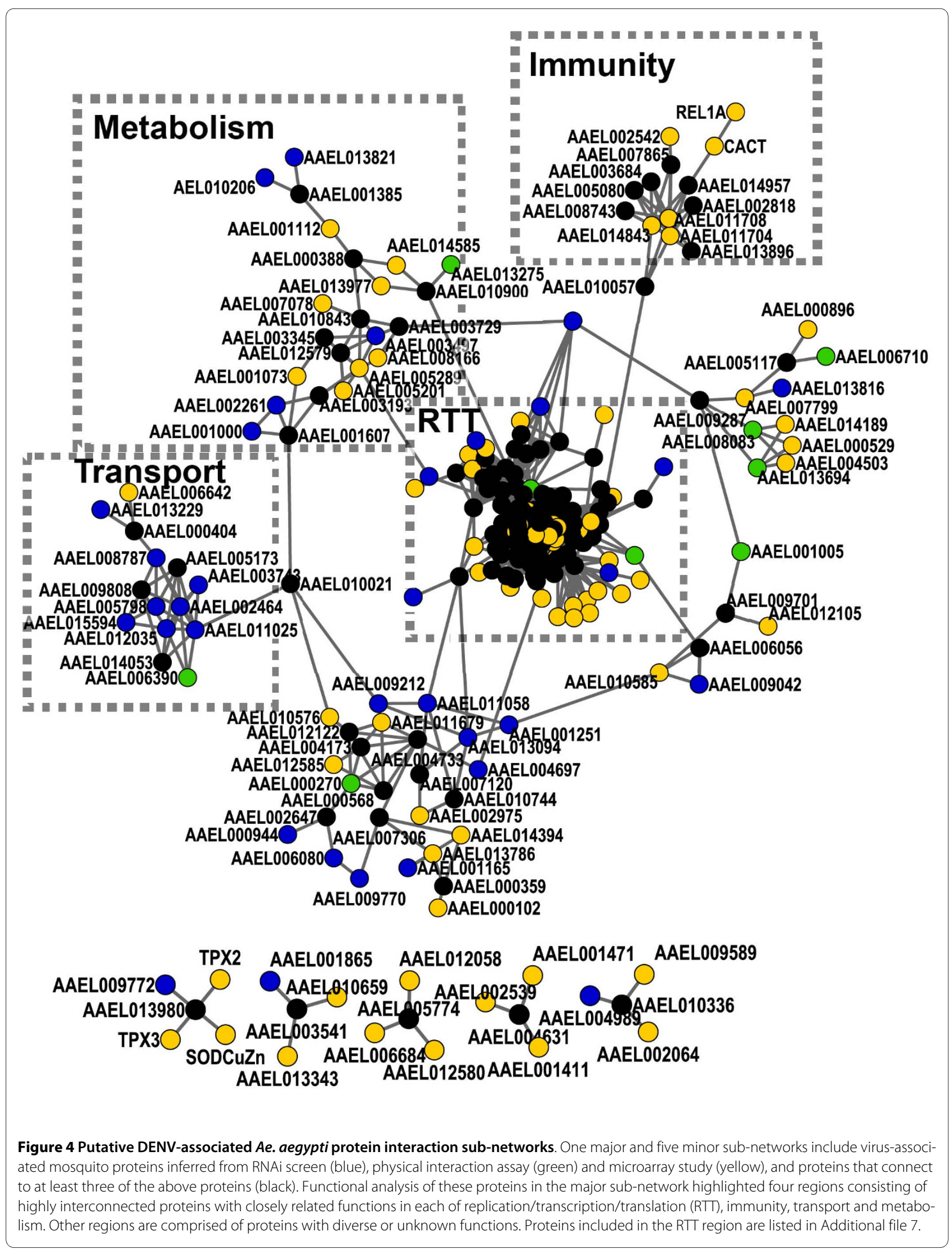


RTT is the most complicated region in the major DENV-associated sub-network, and is comprised of 133 proteins (Fig. 4, Additional file 7). Except for 31 proteins with unknown or diverse functions, the largest proportion (92 out of $133 ; 69.2 \%$ ) of the proteins in this region plays roles in replication, transcription and translation. This can be explained by DENV hijacking cellular proteins to aid in their own replication and transcription and their reliance on host machinery for their translation. As an example, La protein [VectorBase: AAEL003664] was reported to bind to the 3 'and 5 ' end UTR of viral RNA [23]. La protein has also been shown to inhibit viral RNA synthesis in a dose-dependent manner in mosquito Ae. albopictus [24]. As a RNA-binding protein, La is normally located in the nucleus. It has to be redistributed to the cytoplasm of dengue-infected cells to play its role in the viral replication process. It is still unknown how to trigger the export of La protein from the nucleus to the cytoplasm for the binding with the 3'and 5' UTR and regulation of viral RNA synthesis.

Metabolism is another function that was highlighted in one region of the sub-network comprising highly interconnected proteins (Fig. 4). 15 out of 23 proteins (65.2\%) in this region function in metabolism. They include genes that express enzymes to catalyze the reactions in the biosynthesis pathway of L-arginine (AAEL003345), aspartate, phenylalanine (AAEL012579), isoprenoid (AAEL003497) and tetrahydrofolate (AAEL002261). Other proteins in this region are involved in metabolic process of galactose (AAEL001607), phosphorus (AAEL003193), malate (AAEL008166, AAEL001073), lyxose (AAEL013821, AAEL010206), mevalonate pathway I (AAEL005201) and arginase pathway (AAEL005289). As a parasite, DENV depends on the metabolic network of the host cell to provide the energy and macromolecule subunits necessary for their replication. Consequently, host infected by the virus can produce dramatic metabolic alterations. Interestingly, three proteins (AAEL012579, AAEL003497 and AAEL005201) that are related to lipid metabolism were found in this group. Further studies are needed to study the impact of DENV on mosquito metabolism, especially lipid metabolism, and the effects of host metabolic perturbation on DENV replication in mosquitoes.

Immunity is the third function that was highlighted in the major DENV-associated sub-network. A region with highly interconnected proteins consists of the Toll pathway genes and heat shock proteins (Fig. 4). It includes REL1, CACT and three heat shock proteins [VectorBase: AAEL011704, AAEL011708 and AAEL014843]. Highlighting of this Toll pathway-related proteins is consistent with our previous report that the Ae. aegypti Toll pathway can control dengue infection [2]. Previous studies also show that the Onyong-nyong virus induces expression of the heat shock protein cognate $70 \mathrm{~B}$ in $A n$. gambiae, which has an inhibitory effect on the replication of this virus [44]. It will be interesting to explore whether the Toll pathway can regulate the expression of heat shock proteins. There are eight proteins that have never been reported to be associated with dengue infection in this region. Three of them are hypothetical proteins. The others are AAEL008743, AAEL005080, AAEL013896, AAEL002818 and AAEL003684. Among them, AAEL002818 may be related to immune defense. Knockdown of its Drosophila ortholog, U2af50, can induce the expression of immune genes [45].

Fourteen highly interconnected proteins are included in the region with a main function in transport (Fig. 4). Ten of them are involved in ion transport while additional four encode tubulin alpha or beta chains, which can mediate microtubule-based movement. Previous studies found that Sindbis viruses induce dramatic changes in the expression of mosquito genes involved in ion transport processes [46].

In the DENV-associated sub-network, some neighbor proteins interact with a high number of DENV-associated proteins derived from the above three datasets. These proteins are highly like to involve in DENV-mosquito interactions. For example, AAEL009287 is a Ras-related nuclear protein (RAN) involved in the transport of large molecules across the nuclear envelope. In the sub-network, RAN is connected to five putative DENV-associated proteins (Fig. 5). Ribosomal protein p40 [VectorBase: AAEL008083/AAEL013694] is a precursor of the high affinity laminin receptor, a putative receptor for the entry of DENV into host cells [25,47]. While mature laminin receptors are located on the cell surface, p40 has been detected in different compartments of cells, and is suggested to be a shuttle protein between the cytoplasm and the nucleus [48]. Calreticulin [VectorBase: AAEL001005] may bind to the 3'UTR of DENV RNA [42]. It has to be retrotranslocated from endoplasmic reticulum lumen to cytosol for this gene regulatory func-

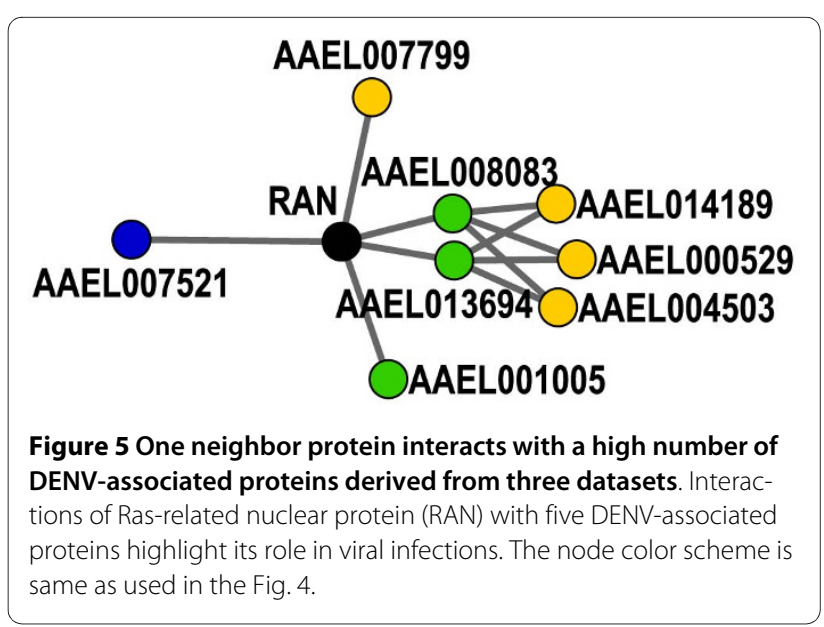


tion [49]. RAN exists in the cell in two nucleotide-bound forms, RanGDP and RanGTP, whose concentration is controlled by the regulator of chromosome condensation (RCC) [VectorBase: AAEL007799]. RAN interacts with importin [VectorBase: AAEL007521] and exportin to change their ability to bind or release cargo molecules for the transport through the nuclear pore. The interactions of RAN with these mosquito proteins suggest that RAN may work together with RCC, importin, and $\mathrm{p} 40$ to redirect viral proteins to the nucleus, and then help the translocation of calreticulin, $\mathrm{La}$, and other RNA-binding proteins to the cytoplasm to facilitate viral genome replication. This hypothesis is supported by the previous studies indicating that RAN was targeted by vesicular stomatitis virus to block transport of RNAs and proteins between the nucleus and cytoplasm of Xenopus laevis oocytes [50]. Further studies are required to elucidate the roles that mosquito RAN protein play in the life cycle of DENV.

\section{Validation of the predicted dengue-mosquito interactions in Ae. aegypti}

To verify the predicted dengue-mosquito interactions, we randomly selected ten mosquito genes from the above DENV-associated mosquito proteins and tested their influences on dengue infection in Ae. aegypti using RNAi knockdown. Three days before mosquitoes were fed with dengue-infected blood, double stranded RNA (dsRNA) of each of these genes was injected into the thorax of mosquitoes. dsRNA of CACT and GFP were injected as positive and negative controls, respectively. Non-injected mosquitoes were used as an additional negative control. Seven days post-infection, mosquito midguts were dissected and the viral titers were measured by plaque assay. As a result, silencing of five out of ten genes (50.0\%) led to a significant reduction of DENV infection in the midgut compared to the GFP dsRNA control (Mann-Whiteney U test, $\mathrm{P}<0.05)$. The anti-dengue effect was also observed in the Cactus dsRNA treatment. There is no significant difference in dengue titer between non-injected and GFP dsRNA injected mosquitoes (Fig. 6 and Additional file 8). These above results support the reliability of our predictions.

Notably, knockdown of a putative tumor suppressor protein [VectorBase: AAEL012515] led to a 13-fold decrease in DENV infection in the mosquito midguts (Fig. 6). The Drosophila ortholog of AAEL012515, TSG101, is the negative regulator of the JAK/STAT pathway and functions as a ubiquitin-protein ligase [51]. The JAK/STAT pathway has been demonstrated to be required for anti-viral defense in Drosophila [52]. In humans, silencing of PIAS2, the protein inhibitor of activated STAT, can also reduce both DENV and WNV infections [20]. The above results strongly suggest that the
JAK/STAT pathway plays an important role in defense against dengue infection in mosquitoes. Our previous studies also show that several important components of this pathway, including DOME [VectorBase: AAEL012471], were up-regulated in response to dengue infection in Ae. aegypti [2]. Thus, the JAK/STAT pathway might play a role in regulation of dengue infection in mosquitoes.

Silencing of the other four genes, AAEL001005, AAEL013989, AAEL005351 and AAEL013275, also significantly reduced the dengue infection in mosquito midguts (Fig. 6). AAEL001005 is a putative mosquito calreticulin protein, whose ortholog in human has been shown to form a complex with 3'UTR in the minusstrand RNA of DENV [42]. Considering that the regulatory sequences involved in viral replication reside in both ends of the flavivirus genome, it is very likely that calreticulin plays a role in the replication of DENV. AAEL013989 is a putative protein translocation complex beta subunit with protein transporter activity. This gene was induced in Ae. aegypti in response to Sindbis virus infection [46]. Silencing of its Drosophila and human ortholog, Sec61 $\beta$, significantly reduced the dengue infection in S2 cell line and HuH-7 cells, respectively [7]. AAEL005351 is Leucine-rich transmembrane protein. Similarly, its Drosophila and human orthologs were proven to be required for dengue infection [7]. Importin beta-1, AAEL013275, is also a protein with transmembrane transporter activity. Previous studies found that dengue virus NS5 is able to interact with the nuclear import receptor importin- $\beta$ although the roles of this interaction is still unclear.

Our RNAi results indicate the notable conservation of required factors between mosquito and human hosts. As described in the above, all the five genes verified here are known to play important roles in interactions of DENV with human host. It appears that common host factors present in human and mosquito, and their interactions with DENV may determine the high efficiency of DENV in entry and replication within these two distantly related animals. In order to maintain the transmission cycle of DENV between mosquito and human, a perfect match in the cellular components and conserved DENV-host interactions may be required between the two host species. Our study makes the first steps towards identification of common pathways that are hijacked by DENV in humans and mosquitoes. A recent study using a genomewide RNA interference screen in D. melanogaster cells found that $51.2 \%$ of the human homolog of dengue associated insect host factors were also required for dengue infection in human [7]. This is consistent with our results that $50.0 \%$ mosquito orthologs of dengue human host factors are required for its infection in mosquito. 


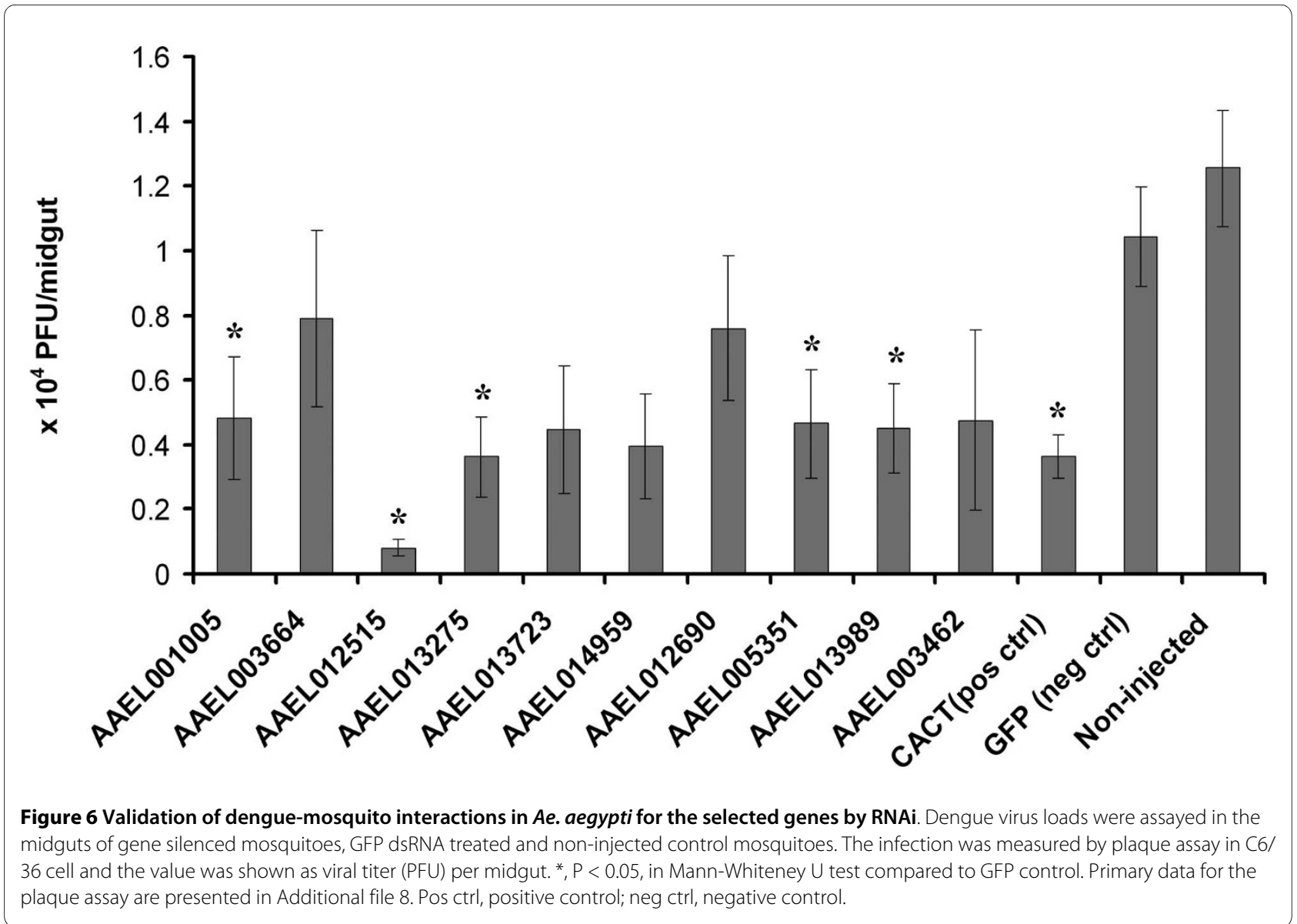

Our studies also show that the protein interaction network is a powerful tool in the study of vector biology. It can not only provide an additional tool for further annotation of vector genome, but can also serve as a novel platform to integrate datasets from different experiments in order to dissect complicated biological processes in insect vectors. By integrating the results from highthroughput assays such as genome-wide RNAi screens, microarrays and yeast two-hybrid system, a protein interaction network can significantly increase prediction accuracy. Moreover, multiple and concurrent signaltransduction pathways operate and are deregulated during the course of vector-pathogen interactions. Such highly interconnected and temporally and spatially regulated signaling pathways can only be dissected by systemlevel approaches. Construction of a protein interaction network can lay a foundation to study the system biology of pathogen-vector interactions, as shown in our integrated analysis of DENV-associated mosquito proteins and mosquito protein interaction network.

As a first step, we used protein interactions data from model organisms to infer a mosquito protein interaction network. In order to understand the unique biological processes in mosquitoes, it is necessary to construct the network using experimental data directly from mosquitoes, such as a high-throughput yeast two-hybrid system. Such an independent mosquito network will allow us to compare and contrast the network's response to dengue infection between humans and mosquitoes. This can reveal unique features of dengue-host interactions in these two species, which will help us understand why DENV causes serious disease in human but no deleterious effects in the mosquito vector.

Arboviruses pass through two different hosts in their life cycle, with great benefit taken from vectors for their mobility, diffusion and evolution. From an implementation standpoint, this also provides unique opportunity for identification of weak linkages in their life history to target using antiviral agents. For example, conserved DENV host factors may be used as targets for anti-dengue agents for blocking dengue transmission in mosquitoes or for pharmacological intervention in humans. With the genome available, insect vectors may be used as an animal model to study the basic biology of arboviruses, and develop novel therapeutics or control strategies. 


\section{Conclusions}

We have developed a mosquito protein interaction network based on large-scale protein interaction datasets in yeast, worm, and fly. The weighted interaction network includes 4,214 mosquito proteins with 10,209 interactions, among which 3,500 proteins are connected into a single interconnected network by 9,719 interactions. This network not only provides a valuable resource for the further annotation of the Ae. aegypti genome, but also makes the first step towards studies of mosquito systems biology. In this regard, we identified a cluster of Toll pathway related protein interactions, and predicted the potential link between the Toll pathway, RNAi pathway and expression of heat shock proteins. We then used datasets from physical interaction assays, RNAi and microarray to infer $A e$ aegypti proteins associated with dengue infection. This led to the identification of 714 putative dengue-associated mosquito proteins. An integrated analysis of these proteins in the network highlighted four regions consisting of highly interconnected proteins with closely related functions in each of replication/transcription/translation (RTT), immunity, transport and metabolism. Finally, by knocking down the predicted DENV-associated mosquito proteins with $\mathrm{RNAi}$, we successfully verified that five of ten candidate genes were required for DENV infection. Our results support the presence of common host requirement of DENV in human and mosquito.

\section{Methods}

\section{Prediction of protein-protein interactions in Ae. aegypti}

We retrieved three high-throughput protein interaction datasets in model organisms along with their confidence scores [12,13,29]. InParanoid was used to determine homology between Ae. aegypti and three model organisms, S. cerevisiae, D. melanogaster, and C. elegans [30]. Proteins $\mathrm{A}$ and $\mathrm{B}$ in $A$ e. aegypti are predicted to interact if their respective orthologs $\mathrm{A}^{\prime}$ and $\mathrm{B}^{\prime}$ are interacting partners in a model organism. We use IP(AA') and IP(BB') to denote InParanoid scores for ortholog pairs $\mathrm{AA}^{\prime}$ and $\mathrm{BB}^{\prime}$, $\mathrm{CS}\left(\mathrm{A}^{\prime} \mathrm{B}^{\prime}\right)$ to indicate confidence values of protein interactions in the model organism. Then, the confidence of predicted interactions in $A$ e. aegypti, $\mathrm{CS}(\mathrm{AB})$ is $\mathrm{CS}\left(\mathrm{A}^{\prime} \mathrm{B}^{\prime}\right) \times$ average of IP(AA') and IP(BB'). If orthologs of proteins $A$ and $B$ in more than one model organism show evidence of interaction, we will have more than one confidence score for A and B. In this case, the maximum value is taken as interaction confidence.

We then retrieved GO terms for all Ae. aegypti proteins based on the TIGR annotation pipeline [53]. GO is comprised of three subcategories, biological process (BP), molecular function (MF), and cellular component (CC). Semantic similarity values were calculated for each predicted interacting protein pair in terms of $\mathrm{BP}, \mathrm{MF}$ and $\mathrm{CC}$ using the SemSim package in BioConductor http:// www.bioconductor.org/packages/2.2/bioc/vignettes/

SemSim/inst/doc/SemSim.pdf. Specifically, Resnik's approach is applied to calculate GO term similarity based on the assumption that the more information two terms share, the more similar they are [54]. The shared information of two terms is indicated by the information content of their parents, defined as the frequency of each term, or any of its children, occurring in an annotated dataset. Less frequently occurring terms are said to be more informative. GO term similarity defines semantic similarity of proteins annotated with these terms. In the case of multiple annotations for a protein, maximum term similarity is taken as the protein similarity [32].

\section{Network analysis of weighted protein interactions}

Global measures of network topology provide quantitative insight into biological systems. We used the igraph package in the $\mathrm{R}$ computing environment to analyze the degree distribution, clustering coefficient, characteristic path length, and diameter of our network. Then, Markov Clustering algorithm (MCL) was applied to partition our mosquito interaction network into densely connected clusters [55], which were displayed and analyzed using the GenePro plugin in Cytoscape [56]. GenePro displays protein clusters as individual nodes interconnected through interacting proteins. Each individual cluster may be expanded to allow detailed analysis in terms of component proteins and their interactions in the context of the full network.

\section{Prediction and analysis of dengue-Ae. aegypti mosquito protein interactions}

Known physical interactions between flavivirus proteins/ RNA and their host proteins were collected by literature search. If an ortholog can be identified in Ae. aegypti for the host protein, then the mosquito protein and corresponding virus protein/RNA are predicted to interact. Both Inparanoid and Ensembl were used for ortholog identification, and only the mosquito protein(s) with the highest Inparanoid score were used for downstream analysis. Similarly, mosquito orthologs were identified for a set of human and Drosophila genes associated with DENV and WNV that were derived from RNA interference screen [20]. Then, a hypergeometric test of GO annotations was used to discover enriched functions in the predicted sets of mosquito proteins using the BinGO plugin [57] in Cytoscape. These proteins were also mapped to the mosquito protein interaction network and the resulting subnetwork was visualized by Cytoscape. The functional classification of mosquito proteins in each module was performed as described previously [53]. The graph density of each functional group or random protein groups with the same size was calculated by graph.density 
function in an $\mathrm{R}$ package igraph http://igraph.sourceforge.net/doc/R/graph.density.html.

\section{Mosquito rearing and cell culture maintenance}

Ae. aegypti mosquitoes of the Rockefeller/UGAL strain were maintained on a sugar solution at $27^{\circ} \mathrm{C}$ and $85 \%$ humidity with a 12-hr light/dark cycle according to standard rearing procedures. The Ae. albopictus cell line C6/ 36 was grown in minimal essential medium (MEM) with $10 \%$ heat inactivated FBS, $1 \%$ L-glutamine, and $1 \%$ nonessential amino acids at $32^{\circ} \mathrm{C}$ with $5 \% \mathrm{CO}_{2}$.

\section{Gene-silencing assays}

DsRNA were synthesized from PCR-amplified gene fragments using the T7 Megascript kit (Ambion). The sequences of the primers are listed in Additional file 9. RNAi-based gene-silencing assays were conducted as previously reported: Approximately $69 \mathrm{nl}$ dsRNAs $(4 \mathrm{mg} /$ $\mathrm{ml}$ ) in water were injected into the thorax of cold-anesthetized 4-day-old female mosquitoes using a nano-injector [2]. Three to four days after injection, mosquitoes were fed on a DENV-2-supplemented blood meal.

\section{DENV-2 infections}

The New Guinea C strain of DENV-2 was propagated in C6/36 cells according to standard conditions. In brief, 0.5 $\mathrm{ml}$ aliquots of virus stock were used to infect $75-\mathrm{cm} 2$ flasks of C6/36 cells at $80 \%$ confluency with a multiplicity of infection (MOI) of 3.5 virus particles/cell. Infected cells were incubated for 5-7 days. Cells were harvested with a cell scraper and lysed to release the virus particles by repeated freezing and thawing in dry $\mathrm{CO}_{2}$ and a $37^{\circ} \mathrm{C}$ water bath. The virus suspension was mixed 1:1 with commercial sheep blood. The blood meal (with $2 \times 10^{7}$ $\mathrm{PFU} / \mathrm{ml} \mathrm{DENV}$ ) was maintained at $37^{\circ} \mathrm{C}$ for $30 \mathrm{~min}$ prior to feeding 6- to 7-day-old mosquitoes http:// www.jove.com/index/Details.stp?ID=220. We confirmed that the virus titer we used resulted in a $90 \%$ infection rate in experimentally infected females by an indirect fluorescent antibody assay performed on head squashes at 14 days after the infectious meal.

\section{Mosquito dissection and Plaque assay}

Seven days after blood meal, mosquitoes were briefly washed in $70 \%$ ethanol, then rinsed in sterile distilled water. The midguts were dissected in sterile PBS and transferred separately to microcentrifuge tubes containing $150 \mathrm{ul}$ of MEM, then homogenized with a Kontes pellet pestle motor in a sterile environment. Each replicate contain 4-5 midguts. At least three independent biological replicate assays were performed for each gene.

\section{Plaque assay}

The virus-containing homogenates were serially diluted and inoculated into C6/36 cells in 24-well plates. After incubation for 5 days at $32^{\circ} \mathrm{C}$ and $5 \% \mathrm{CO} 2$, the plates were assayed for plaque formation by peroxidase immunostaining, using mouse hyperimmune ascitic fluid (MHIAF, specific for DENV-2) and a goat anti-mouse HRP conjugate as the primary and secondary antibody, respectively.

\section{List of abbreviations}

DENV: dengue viruses; JVE: Japanese encephalitis virus; WNV: West Nile; CACT: Cactus; UTR: untranslated regions; HCV: Hepatitis C virus; RNAi: RNA interference; GO: Gene Ontology; BIND: Biomolecular Interaction Network Database; RTT: replication/transcription/ translation; CTL: C-Type Lectins.

\section{Additional material}

Additional file 1 Predicted protein-protein interactions in Ae. aegypti. Additional file $\mathbf{2}$ Semantic similarity and interaction confidence of putative interacting proteins predicted from yeast alone. Protein interactions were binned based on their confidence scores. The average semantic similarity values in each bin were shown for all three GO sub-categories. Percentage of interactions in each bin was shown as a histogram bar.

Additional file $\mathbf{3}$ Clusters in the high-confidence protein interaction network identified by Markov Clustering Algorithm.

Additional file 4 Functional annotation of $\mathbf{7 8}$ clusters comprising more than 10 protein members

Additional file 5 Putative DENV-associated Ae. aegypti proteins based on RNAi screen results.

Additional file 6 Putative DENV-associated Ae. aegypti proteins based on physical interaction data.

Additional file 7 List of highly interconnected proteins in one region of sub-networks with a main function in replication/transcription/ translation (RTT)

Additional file 8 Averaged data from three independent biological replicate plaque assays of the virus titer in the midguts of the gene silenced and GFP dsRNA treated mosquitoes. S.E., standard error; S, significant; NS, Non-significant.

Additional file 9 The prime sequences used for amplification of the target genes. T7 promoter sequence (TAATACGACTCACTATAGGG) is added at the $5^{\prime}$ end of all the primers for synthesis of the dsRNAs.

\section{Authors' contributions}

Conceived and designed the project: XG, ZX. Analyzed the data: XG, ZX and YXI. Performed the experiments: YXU, GB and AP. Contributed reagents/materials/analysis tools: $X G, B G, Y X I$. Wrote the paper: $X G, Z X$. All authors read and approved the final manuscript.

\section{Acknowledgements}

We are grateful to Dr. George Dimopoulos for providing DENV-2 strain and C6/ 36 cell line for this study. We are thankful to the Arbovirus Diseases Branch at the CDC for providing us with the anti-dengue antibodies (Mouse Hyperimmune Ascetic Fluid). We also thank Drs. Suzanne Thiem, C. Titus Brown and Edward D. Walker for editorial assistance and comments on the manuscript. This work was supported by the National Institutes of Health [1R01Al080597 to Z. X.]; and Michigan State University [08-IRGP-1452].

\section{Author Details}

'Department of Entomology and Genetics Program, Michigan State University, East Lansing, Michigan 48824, USA, 2 J Craig Venter Institute, Rockville, MD 20850, USA, ${ }^{3}$ Center for Statistical Training \& Consulting, Michigan State University, East Lansing, Michigan 48824, USA and ${ }^{4}$ Advanced Biomedical Computing Center, SAIC-Frederick Inc, NCI-Frederick, MD 21702, USA

Received: 8 October 2009 Accepted: 16 June 2010

Published: 16 June 2010 


\section{References}

1. Franz AW, Sanchez-Vargas I, Adelman ZN, Blair CD, Beaty BJ, James AA Olson KE: Engineering RNA interference-based resistance to dengue virus type 2 in genetically modified Aedes aegypti. Proc Natl Acad Sci USA 2006, 103(11):4198-4203.

2. Xi Z, Ramirez JL, Dimopoulos G: The Aedes aegypti toll pathway controls dengue virus infection. PLoS Pathog 2008, 4(7):e1000098.

3. Gomez-Machorro C, Bennett KE, del Lourdes Munoz M, Black WCt: Quantitative trait loci affecting dengue midgut infection barriers in an advanced intercross line of Aedes aegypti. Insect Mol Biol 2004, 13(6):637-648

4. Chambers TJ, Hahn CS, Galler R, Rice CM: Flavivirus genome organization, expression, and replication. Annu Rev Microbiol 1990, 44:649-688.

5. Lindenbach BD, Rice CM: Molecular biology of flaviviruses. Adv Virus Res 2003, 59:23-61.

6. Fernandez-Garcia MD, Mazzon M, Jacobs M, Amara A: Pathogenesis of flavivirus infections: using and abusing the host cell. Cell Host Microbe 2009, 5(4):318-328.

7. Sessions OM, Barrows NJ, Souza-Neto JA, Robinson TJ, Hershey CL, Rodgers MA, Ramirez JL, Dimopoulos G, Yang PL, Pearson JL, et al:: Discovery of insect and human dengue virus host factors. Nature 2009, 458(7241):1047-1050

8. Calderwood MA, Venkatesan K, Xing L, Chase MR, Vazquez A, Holthaus AM, Ewence AE, Li N, Hirozane-Kishikawa T, Hill DE, et al:: Epstein-Barr virus and virus human protein interaction maps. Proc Natl Acad Sci USA 2007, 104(18):7606-7611.

9. Forst CV: Host-pathogen systems biology. Drug Discov Today 2006, 11(56):220-227.

10. de Chassey B, Navratil V, Tafforeau L, Hiet MS, Aublin-Gex A, Agaugue S, Meiffren G, Pradezynski F, Faria BF, Chantier T, et al:: Hepatitis C virus infection protein network. Mol Syst Bio/ 2008, 4:230.

11. Dyer MD, Murali TM, Sobral BW: The landscape of human proteins interacting with viruses and other pathogens. PLoS Pathog 2008, 4(2):e32.

12. Giot L, Bader JS, Brouwer C, Chaudhuri A, Kuang B, Li Y, Hao YL, Ooi CE, Godwin B, Vitols $E$, et al: A protein interaction map of Drosophila melanogaster. Science 2003, 302(5651):1727-1736.

13. Li S, Armstrong CM, Bertin N, Ge H, Milstein S, Boxem M, Vidalain PO, Han JD, Chesneau A, Hao T, et al:: A map of the interactome network of the metazoan C. elegans. Science 2004, 303(5657):540-543.

14. Uetz P, Giot L, Cagney G, Mansfield TA, Judson RS, Knight JR, Lockshon D, Narayan V, Srinivasan M, Pochart P, et al.: A comprehensive analysis of protein-protein interactions in Saccharomyces cerevisiae. Nature 2000, 403(6770):623-627.

15. Lehner B, Fraser AG: A first-draft human protein-interaction map. Genome Biol 2004, 5(9):R63.

16. Brown KR, Jurisica I: Online predicted human interaction database. Bioinformatics 2005, 21(9):2076-2082.

17. Geisler-Lee J, O'Toole N, Ammar R, Provart NJ, Millar AH, Geisler M: A predicted interactome for Arabidopsis. Plant Physiol 2007 145(2):317-329

18. Ideker T, Lauffenburger D: Building with a scaffold: emerging strategies for high- to low-level cellular modeling. Trends Biotechno/ 2003, 21(6):255-262

19. Uetz P, Dong YA, Zeretzke C, Atzler C, Baiker A, Berger B, Rajagopala SV, Roupelieva M, Rose D, Fossum E, et al:: Herpesviral protein networks and their interaction with the human proteome. Science 2006 311(5758):239-242

20. Krishnan MN, Ng A, Sukumaran B, Gilfoy FD, Uchil PD, Sultana H, Brass AL, Adametz R, Tsui M, Qian F, et al:: RNA interference screen for human genes associated with West Nile virus infection. Nature 2008, 455(7210):242-245

21. Ren J, Ding T, Zhang W, Song J, Ma W: Does Japanese encephalitis virus share the same cellular receptor with other mosquito-borne flaviviruses on the C6/36 mosquito cells? Virol J 2007, 4:83.

22. Chu JJ, Leong PW, Ng ML: Characterization of plasma membraneassociated proteins from Aedes albopictus mosquito $(C 6 / 36)$ cells that mediate West Nile virus binding and infection. Virology 2005, 339(2):249-260

23. Garcia-Montalvo BM, Medina F, del Angel RM: La protein binds to NS5 and NS3 and to the 5 ' and 3 ' ends of Dengue 4 virus RNA. Virus Res 2004, 102(2):141-150.
24. Yocupicio-Monroy M, Padmanabhan R, Medina F, del Angel RM: Mosquito La protein binds to the 3 ' untranslated region of the positive and negative polarity dengue virus RNAs and relocates to the cytoplasm of infected cells. Virology 2007, 357(1):29-40.

25. Thepparit C, Smith DR: Serotype-specific entry of dengue virus into liver cells: identification of the 37-kilodalton/67-kilodalton high-affinity laminin receptor as a dengue virus serotype 1 receptor. J Virol 2004 78(22):12647-12656.

26. Sakoonwatanyoo P, Boonsanay V, Smith DR: Growth and production of the dengue virus in C6/36 cells and identification of a laminin-binding protein as a candidate serotype 3 and 4 receptor protein. Intervirology 2006, 49(3):161-172

27. Reyes-Del Valle J, Chavez-Salinas S, Medina F, Del Angel RM: Heat shock protein 90 and heat shock protein 70 are components of dengue virus receptor complex in human cells. J Virol 2005, 79(8):4557-4567.

28. Salas-Benito J, Reyes-Del Valle J, Salas-Benito M, Ceballos-Olvera I, Mosso C, del Angel RM: Evidence that the 45-kD glycoprotein, part of a putative dengue virus receptor complex in the mosquito cell line C6/ 36, is a heat-shock related protein. Am J Trop Med Hyg 2007, 77(2):283-290.

29. Bader JS, Chaudhuri A, Rothberg JM, Chant J: Gaining confidence in high-throughput protein interaction networks. Nat Biotechnol 2004 22(1):78-85

30. Remm M, Storm CE, Sonnhammer EL: Automatic clustering of orthologs and in-paralogs from pairwise species comparisons. J Mol Biol 2001 314(5):1041-1052.

31. Lu LJ, Xia Y, Paccanaro A, Yu H, Gerstein M: Assessing the limits of genomic data integration for predicting protein networks. Genome Res 2005, 15(7):945-953.

32. Guo X, Liu R, Shriver CD, Hu H, Liebman MN: Assessing semantic similarity measures for the characterization of human regulatory pathways. Bioinformatics 2006, 22(8):967-973.

33. Brohee $\mathrm{S}$, van Helden J: Evaluation of clustering algorithms for proteinprotein interaction networks. BMC Bioinformatics 2006, 7:488.

34. Shin SW, Kokoza V, Bian G, Cheon HM, Kim YJ, Raikhel AS: REL1, a homologue of Drosophila dorsal, regulates toll antifungal immune pathway in the female mosquito Aedes aegypti. J Bio/ Chem 2005 280(16):16499-16507.

35. Christophides GK, Vlachou D, Kafatos FC: Comparative and functiona genomics of the innate immune system in the malaria vector Anopheles gambiae. Immunol Rev 2004, 198:127-148.

36. Hoffmann JA: The immune response of Drosophila. Nature 2003 426(6962):33-38

37. Lemaitre B, Nicolas E, Michaut L, Reichhart JM, Hoffmann JA: The dorsoventral regulatory gene cassette spatzle/Toll/cactus controls the potent antifungal response in Drosophila adults. Cell 1996, 86(6):973-983.

38. Ishizuka A, Siomi MC, Siomi H: A Drosophila fragile $X$ protein interacts with components of RNAi and ribosomal proteins. Genes Dev 2002, 16(19):2497-2508

39. Waterhouse RM, Kriventseva EV, Meister S, Xi Z, Alvarez KS, Bartholomay LC, Barillas-Mury C, Bian G, Blandin S, Christensen BM, et al:: Evolutionary dynamics of immune-related genes and pathways in disease-vector mosquitoes. Science 2007, 316(5832):1738-1743

40. Modis Y, Ogata S, Clements D, Harrison SC: A ligand-binding pocket in the dengue virus envelope glycoprotein. Proc Natl Acad Sci USA 2003, 100(12):6986-6991.

41. Chee HY, AbuBakar S: Identification of a $48 \mathrm{kDa}$ tubulin or tubulin-like C6/36 mosquito cells protein that binds dengue virus 2 using mass spectrometry. Biochem Biophys Res Commun 2004, 320(1):11-17.

42. Yocupicio-Monroy RM, Medina F, Reyes-del Valle J, del Angel RM: Cellular proteins from human monocytes bind to dengue 4 virus minus-strand 3' untranslated region RNA. J Virol 2003, 77(5):3067-3076.

43. Shafee $N$, AbuBakar S: Effects of Dengue 2 Virus Inoculation on. Toxorhynchites splendens Larvae. Journal of Entomology 2006, 3(1):89-94.

44. Sim C, Hong YS, Tsetsarkin KA, Vanlandingham DL, Higgs S, Collins FH: Anopheles gambiae heat shock protein cognate 70B impedes o'nyongnyong virus replication. BMC Genomics 2007, 8:231.

45. Foley $\mathrm{E}, \mathrm{O}$ 'Farrell $\mathrm{PH}$ : Functional dissection of an innate immune response by a genome-wide RNAi screen. PLOS Biol 2004, 2(8):E203.

46. Sanders HR, Foy BD, Evans AM, Ross LS, Beaty BJ, Olson KE, Gill SS: Sindbis virus induces transport processes and alters expression of innate 
immunity pathway genes in the midgut of the disease vector, Aedes aegypti. Insect Biochem Mol Biol 2005, 35(11):1293-1307.

47. Bogachek MV, Protopopova EV, Loktev VB, Zaitsev BN, Favre M, Sekatskii SK, Dietler G: Immunochemical and single molecule force spectroscopy studies of specific interaction between the laminin binding protein and the West Nile virus surface glycoprotein E domain II. JMol Recognit 2008, 21(1):55-62.

48. Salama RH, Muramatsu H, Zou K, Inui T, Kimura T, Muramatsu T: Midkine binds to $37-\mathrm{kDa}$ laminin binding protein precursor, leading to nuclear transport of the complex. Exp Cell Res 2001, 270(1):13-20.

49. Afshar N, Black BE, Paschal BM: Retrotranslocation of the chaperone calreticulin from the endoplasmic reticulum lumen to the cytosol. $\mathrm{Mol}$ Cell Biol 2005, 25(20):8844-8853.

50. Petersen JM, Her LS, Varvel V, Lund E, Dahlberg JE: The matrix protein of vesicular stomatitis virus inhibits nucleocytoplasmic transport when it is in the nucleus and associated with nuclear pore complexes. Mol Cell Biol 2000, 20(22):8590-8601.

51. Muller P, Kuttenkeuler D, Gesellchen V, Zeidler MP, Boutros M: Identification of JAK/STAT signalling components by genome-wide RNA interference. Nature 2005, 436(7052):871-875.

52. Dostert C, Jouanguy E, Irving P, Troxler L, Galiana-Arnoux D, Hetru C, Hoffmann JA, Imler JL: The Jak-STAT signaling pathway is required but not sufficient for the antiviral response of drosophila. Nat Immunol 2005, 6(9):946-953.

53. Nene V, Wortman JR, Lawson D, Haas B, Kodira C, Tu ZJ, Loftus B, Xi Z, Megy K, Grabherr M, et al:: Genome sequence of Aedes aegypti, a major arbovirus vector. Science 2007, 316(5832):1718-1723.

54. Resnik P: Semantic similarity in a taxonomy: an information-based measure and its application to problems of ambiguity in natural language. J Artif Intel Res 1999, 11:95-130.

55. Enright AJ, Van Dongen S, Ouzounis CA: An efficient algorithm for largescale detection of protein families. Nucleic Acids Res 2002, 30(7):1575-1584

56. Vlasblom J, Wu S, Pu S, Superina M, Liu G, Orsi C, Wodak SJ: GenePro: a Cytoscape plug-in for advanced visualization and analysis of interaction networks. Bioinformatics 2006, 22(17):2178-2179.

57. Maere S, Heymans K, Kuiper M: BiNGO: a Cytoscape plugin to assess overrepresentation of gene ontology categories in biological networks. Bioinformatics 2005, 21(16):3448-3449.

doi: 10.1186/1471-2164-11-380

Cite this article as: Guo et al, Response of the mosquito protein interaction network to dengue infection BMC Genomics 2010, 11:380
Submit your next manuscript to BioMed Central and take full advantage of:

- Convenient online submission

- Thorough peer review

- No space constraints or color figure charges

- Immediate publication on acceptance

- Inclusion in PubMed, CAS, Scopus and Google Scholar

- Research which is freely available for redistribution

Submit your manuscript at www.biomedcentral.com/submit
C Biomed Central 